\title{
Improved Non Mutual Information based Multi-path Time Delay Estimation
}

\author{
S. Radhika ${ }^{1 *}$ and Sivabalan Arumugam ${ }^{2}$ \\ 'Faculty of Electrical and Electronics Engineering, Sathyabama University, \\ Chennai,Tamil Nadu, India; radhikachandru79@gmail.com \\ ${ }^{2}$ NEC Mobile networks Excellence Centre, Chennai, Tamil Nadu, India
}

\begin{abstract}
Estimation of time delay associated with a given signal is always an interesting and useful problem in many applications like radar, sonar, tele-conferencing, wireless networks. It is important because the results obtained from the time delay estimators are used for further processing. Finding the time delay especially in noisy environment is always complicated and challenging and an accurate delay estimator is inevitable. Most of the time delay estimation techniques use second order statistics. In this paper, an improved new delay estimator based on information theory criteria to work in noisy environment is proposed. Simulation results validate the improvement in the time delay estimation even in very low signal to noise ratio conditions.
\end{abstract}

Keywords: Correlation, Joint Entropy, Mutual Information, Non Mutual Information, Time Delay Estimation

\section{Introduction}

Multipath is a phenomena which regularly occurs in radar, sonar, reverberation environment. Their occurrence is usually undesirable. However it necessary to find the parameters of the multipath signal such as attenuation, time delay etc., which finds application in acoustic echo cancellation ${ }^{13}$, seismography, underwater acoustics, source localization, tracking of cameras. Multipath is said to occur when the signal has more than one paths. The paths include direct path and as well as the attenuated, delayed version of the direct path signals and hence multipath. In case of acoustic environment, these extra paths are called echoes or reflection. All these echo paths are undesirable therefore acoustic echo cancellers are used to solve this problem. The conventional echo cancellers uses one single long filter which has poor convergence and high complexity.

$\operatorname{In}^{11,12,20}$ it is said that parallel recursive filter can be used to reduce the computational complexity and can improve the convergence rate. $\mathrm{In}^{21}$, it is said that the nature of the input has an impact on the convergence rate of the adaptive filters. $\mathrm{In}^{13}$, the acoustic echo is modeled as a multipath signal in order to build an adaptive multiple sub filters. In $^{22}$ a time delay estimator based on the multiple path model is proposed. Also the performance of the estimator is evaluated in terms of number of multiple path and various noise conditions.

$\mathrm{In}^{19}$, the time delay estimation is used for system identification so that the model structure with a certain order which when adjusted for an identification dataset, represents the true system. Therefore in this paper time delay estimation is used to build a model for the acoustic echo channel which can be used to develop less complex adaptive filter. In order to realize the echo paths, accurate time delay measurement is necessary.

Time delay estimators find the relative delay in the time of the signals received at the sensors ends. Time delay estimation algorithms usually make use of correlation. The most widely used method is the generalized cross correlation method by Knapp and Carter ${ }^{2}$. Some of the variants of it includes auto correlation method ${ }^{14}$, PHAT, SCOT ${ }^{15}$, MCCC $^{16}$. All these are based on second order statistics. Moreover they perform well when the noise level is low.

*Author for correspondence 
In this paper, time delay estimation is approached from the information theory point of view. Entropy is a measure of uncertainty associated with the random variables. Higher Order Statistics (HOS) can say about the dependencies more than second order statistics. Hence entropy, which is part of HOS, is used in this paper for time delay estimation.

$\mathrm{In}^{27}$, the advantages of higher order statistics for TDE is discussed and it concluded that HOS estimation method has many advantages such as more accuracy, small error etc. $\mathrm{In}^{24}$ the significance of information theory criteria and the usefulness of nearest neighbor with respect to medical application is discussed. $\mathrm{In}^{25}$, an information theory based TDE method which is an indirectly encapsulating Higher Order Statistics (HOS) is used. Also in this paper generalized Gaussian distribution that allows evaluating the problem under a larger set of speech-shaped distributions, ranging from Gaussian to Laplacian and Gamma is proposed. The results indicate that TDE based on the specific criterion is independent of the underlying assumption for the distribution of the source, for the same covariance matrix.

$\mathrm{In}^{3}$ it has been proved that multi channel cross correlation method is equal to joint entropy and it far exceeds MCCC in performance when real reverberant environment is taken. $\operatorname{In}^{23}$ a TDE based on minimum entropy for non Gaussian signal is compared with minimum dispersion and the equivalence between them is proved. Also in ${ }^{27}$ a TDE for laplace distribution using minimum entropy is proposed.

In $^{5,6,26}$ it has been said that mutual information between the signals can be used for TDE. $\operatorname{In}^{8}$ a comparison between the cross correlation and mutual information based time delay estimation is made and an improved time delay estimation based on spreading of information to neighbor hood samples is discussed.

$\operatorname{In}^{17}$ it has been said that joint entropy is made from mutual and non mutual information. Also in ${ }^{17}$ a time delay estimation is proposed using non mutual information and a comparison of TDE using joint entropy, mutual information and non mutual information is made and it is concluded that all the above perform well in background noise and non mutual information based TDE outperform the joint and mutual information under reverberant condition.

Thus from the above it is found that the non mutual information based TDE is very apt for noisy and multipath acoustic environment. In this paper we propose a mathematical frame work that estimates the time delay even in adverse noisy condition. It makes use of the spreading of information among neighboring samples. Hence non mutual information criteria by taking into consideration of the neighboring samples is used to give more accurate time delay. The upgraded algorithm is justified by simulation. The source is assumed to be Gaussian.

The remaining part of this paper is organized as follows. Section II deals with the information theory criteria, Section III describes the signal model and the proposed estimator is discussed in section IV. Simulation results are described in Section V \& conclusion and future work is illustrated in section VI.

\section{Information Theory Concepts}

Most of the time delay estimation depends on second order statistics. The dependency of the variables can be studied in a better way if higher order statistics is employed. The following are the different HOS based on information theory criteria for time delay estimation

\subsection{Entropy}

Entropy is a measure of the uncertainty associated with the random variables. If $\mathrm{x}$ is a continuous random variable and $\mathrm{p}(\mathrm{x})$ is the probability density function then the entropy is defined mathematically as

$$
H(x)=-\int p(x) \log p(x) d x
$$

If $\mathrm{x}$ is a discrete random variable with $\mathrm{pdf}$ as $\mathrm{p}(\mathrm{x})$ then the entropy is defined as

$$
\mathrm{H}(\mathrm{x})=-\sum_{N} \mathrm{p}(\mathrm{x}) \log \mathrm{p}(\mathrm{x})
$$

\subsection{Joint Entropy}

If there are $\mathrm{M}$ random variables $\mathrm{X}=[\mathrm{x} 1, \mathrm{x} 2, \mathrm{x} 3, \mathrm{x} 4 \ldots \mathrm{xM}] \mathrm{T}$ each with probability density function as $\mathrm{p}(\mathrm{xi})$ for $\mathrm{i}=1,2,3, \ldots \mathrm{M}$ and if the random variables belong to a multivariate normal distribution with mean 0 and variance $\sigma 2 x i$ then the joint entropy is given ${ }^{3}$

$$
H(\mathrm{x})=\frac{1}{2} \ln \left\{(2 \pi e)^{M} \operatorname{det}(R)\right\}
$$

where, $\mathrm{R}=$ covariance matrix given by

$$
R=E\{X X T\}
$$

Therefore, for a multivariate normally distributed random variables. If $\mathrm{x}(\mathrm{n}, \mathrm{m})$ is a vector formed by the random variables $\mathrm{x}_{1}, \mathrm{x}_{2}, \mathrm{x}_{\mathrm{M}}$ then 


$$
\text { time delay }=\arg \min _{m}(\mathrm{H}(\mathrm{x}(\mathrm{n}, \mathrm{m})))
$$

\subsection{Mutual Information}

Mutual information is one other criterion which tells about the information that two random variables have common between them. If there are two random variables $\mathrm{x}_{1}$ and $\mathrm{x}_{2}$ with a joint

Probability density function $\mathrm{p}\left(\mathrm{x}_{1}, \mathrm{x}_{2}\right)$ and marginal probability density functions as $\mathrm{p}\left(\mathrm{x}_{1}\right)$ and $\mathrm{p}\left(\mathrm{x}_{2}\right)$. The mutual information $\mathrm{I}\left(\mathrm{x}_{1} ; \mathrm{x}_{2}\right)$ is given ${ }^{4}$

$$
\begin{aligned}
\mathrm{I}(\mathrm{x} 1 ; \mathrm{x} 2) & =\sum_{\mathrm{x} 1, \mathrm{x} 2} \mathrm{p}(\mathrm{x} 1, \mathrm{x} 2) \log \frac{\mathrm{p}(\mathrm{x} 1, \mathrm{x} 2)}{\mathrm{p}(\mathrm{x} 1) \mathrm{p}(\mathrm{x} 2)} \\
& =\mathrm{H}(\mathrm{x} 1)+\mathrm{H}(\mathrm{x} 2)-\mathrm{H}(\mathrm{x} 1, \mathrm{x} 2)
\end{aligned}
$$

If the random variables follow Gaussian distribution with zero mean and variance oxi then the mutual information is given ${ }^{4}$

$$
I=-\frac{1}{2} \ln \operatorname{det}(\mathrm{R})\left(\frac{\mathrm{R}_{11}}{\mathrm{R}_{22}}\right)
$$

where, $\mathrm{R}$ is the covariance matrix and $\mathrm{R} 11$ and $\mathrm{R} 22$ are time shifted independent variables.

The mutual information for a multivariate normally distributed random variables is given ${ }^{18}$ as

$$
\begin{aligned}
\mathrm{IM}(\mathrm{x} 1 ; \mathrm{x} 2 ; \ldots \mathrm{xM})= & (\mathrm{H}(\mathrm{x} 1)+\mathrm{H}(\mathrm{x} 2) \ldots+\mathrm{H}(\mathrm{xM}))-\ldots \\
& +(-1) \mathrm{M}-1 \mathrm{H}(\mathrm{x} 1, \mathrm{x} 2, \ldots \mathrm{xM})
\end{aligned}
$$

If the random variables follow Gaussian distribution then the mutual information is given ${ }^{8}$

$$
\mathrm{IM}=-\frac{1}{2} \ln \operatorname{det}(\mathrm{R})\left(\frac{\operatorname{det} \mathrm{R}_{11}}{\operatorname{det} \mathrm{R}_{22}}\right)
$$

where, $\mathrm{R}$ is the covariance matrix of multivariate normal random variables given by

$$
\mathrm{R}=[\mathrm{x} 1, \mathrm{x} 2, \mathrm{x} 3, \ldots \mathrm{xM}] *[\mathrm{x} 1, \mathrm{x} 2, \mathrm{x} 3, \ldots \mathrm{xM}] \mathrm{T}
$$

Therefore the time delay is given by that value of $\mathrm{m}$ that maximizes the mutual information.

$$
\begin{aligned}
& \text { time delay }=\arg \max (\mathrm{MI}(\mathrm{x}(\mathrm{n}, \mathrm{m}))) \\
& m
\end{aligned}
$$

\subsection{Non Mutual Information}

From ${ }^{17}$ it is said that the total information is given as joint entropy which consists of the mutual information which is the information shared by all the signals and the non mutual information which is the information not shared by the signals. Therefore the non mutual information is obtained as

Non mutual information $=$ joint entropy

$$
\text { - mutual information }
$$

\section{Signal Model}

We take up the case of a single channel environment where a loudspeaker and a microphone are placed in a room with a distance of $d$ meters between them. The sound received at the sensor end has multiple paths in addition to direct path due to reflections from various objects, ceiling etc. If the room is non reverberant, then the signal model can be written ${ }^{9}$

$$
y(n)=\sum_{i=0}^{M} x(n-D i)+w(n)
$$

In the model described by (13) the received signals is said to have difference only in delay. Also in the conventional room acoustic reverberant environment, the impulse response is said to be very long which makes the convergence very slow. Therefore as in $\operatorname{paper}^{10}$ the entire impulse response is segmented into different multipaths each has filtering characteristics. Moreover if the impulse response of the room is simulated using image source method, then the spectral analysis concludes that it has low pass filtering nature. Also the signal in the extra paths has attenuation and delay due to reflections from the walls, floor, materials etc. Thus the signal from the loudspeaker to microphone can be modeled as the sum, delayed, attenuated and filtered version of the input signal. Therefore the model of the signal used in analysis can be written ${ }^{10}$.

$$
\mathrm{y}(\mathrm{n})=\mathrm{gi}(\mathrm{h}(\mathrm{n}) * \mathrm{x}(\mathrm{n}))+\mathrm{w}(\mathrm{n})
$$

where, $*$ represents convolution operation.

$$
y(n)=g i \sum_{i=0}^{M} h(n) x(n-D i)+w(n)
$$

where,

$\mathrm{D}_{0}$ is the time taken by the signal to reach the microphone which corresponds to the direct path,

$\mathrm{D}_{1}, \mathrm{D}_{2}, \mathrm{D}_{3}, \ldots \mathrm{D}_{\mathrm{M}}$ are the delays of the multipath signal, $\mathrm{y}(\mathrm{n})$ is the received output signal. 
$\mathrm{w}(\mathrm{n})$ is the random noise having zero mean and unity variance which is uncorrelated with the source signal.

$h(n)$ is the impulse response of a low pass filter.

$\mathrm{x}(\mathrm{n})$ is the input signal.

$M$ is the number of multipaths.

gi's are the attenuation constants which depends on the parameters of the room.

\section{Proposed Estimator}

In case of simple multipath environment there will be only one delay present in between the direct signal and the delayed signal. Thus the information contained in kth sample of $\mathrm{x} 1$ is dependent on the information that corresponds to $\mathrm{k}$-Dith sample of $\mathrm{x} 2$. But the signal model consists of a filter in each path. Therefore the information contained in kth sample of $\mathrm{x} 1$ is spread to the neighbors of $k$-Dith sample of $x 2$ due to the convolution operation done to represent the multipath signals. Also it suffers from the same drawback of the cross correlation and auto correlation methods. So normal mutual information is not a representative for time delay estimation. From $^{8}$ it is found that the (9) can used to find time delay which takes $\mathrm{N}$ neighboring samples.

From $^{17}$ it is found that direct use of mutual information cannot identify true time delay because of the convolution operation. The solution is to find the mutual information along with the neighborhood samples also. From ${ }^{18}$ non mutual information which is the complement of the former can find the true time delay. From (12), the difference between joint entropy and mutual information gives the time delay estimation. Due to the spreading of mutual information with $\mathrm{N}$ neighboring samples, even though non mutual information is complement of the mutual information, it also suffers from the same problem as mutual information. Therefore an improved non mutual information based TDE is also found by taking the neighboring samples also is proposed.

Assume there are M multiple path present, then the vector

$$
\mathrm{x}(\mathrm{n}, \mathrm{m})=\triangleq[\mathrm{x} 1(\mathrm{n}, \mathrm{m}) \mathrm{x} 2(\mathrm{n}, \mathrm{m}) \ldots \mathrm{xM}(\mathrm{n}, \mathrm{m})] \mathrm{T}
$$

is formed by taking all the signals. The joint entropy is given as in (3). From ${ }^{8}$ the mutual information is given by (8).

By substituting the equations (3) and (8) in (12) we get the modified non mutual information. It can be found by taking the joint entropy and mutual information of $\mathrm{N}$ neighboring samples. Thus

$$
\begin{aligned}
\operatorname{MNMI}(\mathrm{x}(\mathrm{n}, \mathrm{m}))= & \mathrm{H}(\mathrm{x}(\mathrm{n}, \mathrm{m})-\mathrm{IM}(\mathrm{x}(\mathrm{n}, \mathrm{m})) \\
\operatorname{MNNI}(\mathrm{x}(\mathrm{n}, \mathrm{m}))= & \mathrm{H}(\mathrm{x}(\mathrm{n}, \mathrm{m}))-[\mathrm{H}(\mathrm{x} 1(\mathrm{k})) \\
& +\mathrm{H}(\mathrm{x} 1(\mathrm{k}+1))+\mathrm{H}(\mathrm{x} 1(\mathrm{k}+2)) \\
& +\ldots+\mathrm{H}(\mathrm{x} 1(\mathrm{k}+\mathrm{N}))+\mathrm{H}(\mathrm{x} 2(\mathrm{k})) \\
& +\mathrm{H}(\mathrm{x} 2(\mathrm{k}+1))+\mathrm{H}(\mathrm{x} 2(\mathrm{k}+2)) \\
& +\ldots+\mathrm{H}(\mathrm{x} 2(\mathrm{k}+\mathrm{N}))+\mathrm{H}(\mathrm{xM}(\mathrm{k})) \\
& +\mathrm{H}(\mathrm{xM}(\mathrm{k}+1))+\mathrm{H}(\mathrm{xM}(\mathrm{k}+2)) \\
& +\ldots+\mathrm{H}(\mathrm{xM}(\mathrm{k}+\mathrm{N}))-\mathrm{H}(\mathrm{x} 1(\mathrm{k}), \\
& \mathrm{x} 1(\mathrm{k}+1), \mathrm{x} 1(\mathrm{k}+2), \ldots, \mathrm{x} 1(\mathrm{k}+\mathrm{N}), \\
& \mathrm{x} 2(\mathrm{k}), \mathrm{x} 2(\mathrm{k}+1), \mathrm{x} 2(\mathrm{k}+2), \ldots, \\
& \mathrm{x} 2(\mathrm{k}+\mathrm{N}), \mathrm{xM}(\mathrm{k}), \mathrm{xM}(\mathrm{k}+1), \\
& \mathrm{xM}(\mathrm{k}+2), \mathrm{xM}(\mathrm{k}+\mathrm{N}))]
\end{aligned}
$$

Accordingly with $\mathrm{M}$ random variables with $\mathrm{N}$ neighboring samples, that value of $\mathrm{m}$ that minimizes (17) gives the time delay. Therefore

$$
\text { time delay }=\arg \min _{m}(\operatorname{MNMI}(\mathrm{x}(\mathrm{n}, \mathrm{m})))
$$

\section{Simulation}

In this section the performance of the modified non mutual information based time delay estimator is evaluated.

The simulation was carried out by taking the model of loudspeaker enclosed microphone system. The number of paths are taken to be 3 including direct path. Each path is assumed to be a low pass filter of order 12. The attenuation for the paths except direct path were chosen to have any value between 0.5 to 1 . The speech signal of $1 \mathrm{sec}$ duration with sampling frequency $8 \mathrm{kHz}$ is taken. Integer delays are chosen for simulation purpose. The delays for the paths are chosen to be 1600 samples, and 3200 samples for a delay of $0.2 \mathrm{sec}$ and $0.4 \mathrm{sec}$. The signal for each path is obtained by convoluting the speech signal and impulse response of low pass filter of order 12. Also Gaussian noise is added to have an appreciable signal to noise ratio. The simulation results are averaged over 50 independent runs and the length of the sequence was taken to be 8000 .

The performance of the estimator is evaluated in terms of accuracy percentage and root mean square error. Accuracy percentage is defined as the no of times the delay is estimated correctly divided by the total no of trials ${ }^{13}$. 


\section{Accuracy Percentage \\ $=\frac{\text { no of time } \operatorname{sindividual} \text { delay is estimated correctly }}{}$ (no of trials * no of delay units)}

A plot of Accuracy percentage and SNR is shown in Figure 1 . The simulation is carried out by taking 2 neighbouring samples. Obviously $\mathrm{N}=0$ is the NMI method. From the graph it is found that at moderate to high SNR, the AP is same for both NMI and MNMI methods. Hence we concentrate only with the low signal to noise ratio, At SNR less than $-10 \mathrm{db}$, the performance of MNMI is slightly better than NMI method. This proves that the MNMI method can able to have more accurate time delay estimation especially in low SNR.

Root Mean Square Error (RMSE) is the other measure used to evaluate the performance of the algorithm. It is defines as the square root of the average value of the difference in the actual and estimated delays. If the actual delays are $\mathrm{d} 1$ and $\mathrm{d} 2$, and the estimated delays are $\widehat{d 1}$ and $\widehat{d 2}$ then the RMSE is found as

$$
R M S E=\sqrt{(d 1-\widehat{d}) 2+(d 2-\widehat{d 2}) 2}
$$

The lower the value of RMSE, better the estimator performance. Hence the simulation is carried out to find the impact of the spreading of information to the neighboring samples. Therfore, RMSE is found for different lengths of the neighbor samples. The simulation is carried out for a $\mathrm{SNR}=-20 \mathrm{db}$. Also an observation length up to 5 neighboring samples is taken. A plot of RMSE and observation length shows that as the observation length is increased, the RMSE value decreases which shows that there is improvement in TDE with increase in the number of neighbor samples. The value of RMSE for NMI corresponds to the point where $\mathrm{N}=0$. The effect is more profound near the boundary than the regions far apart. Thus observation length has a great impact on the time delay accuracy (Figure 2).

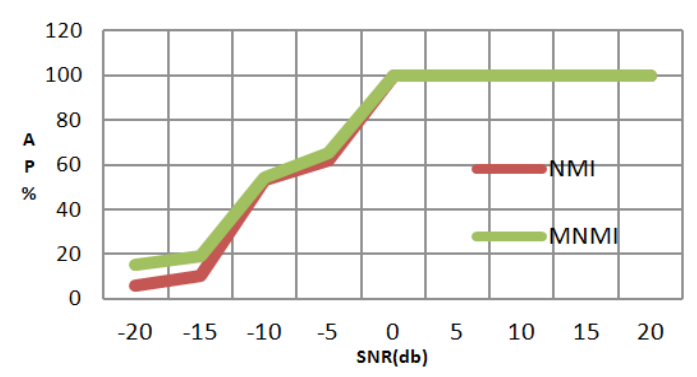

Figure 1. Accuracy Percentage Vs SNR(db) for $\mathrm{N}=2$.

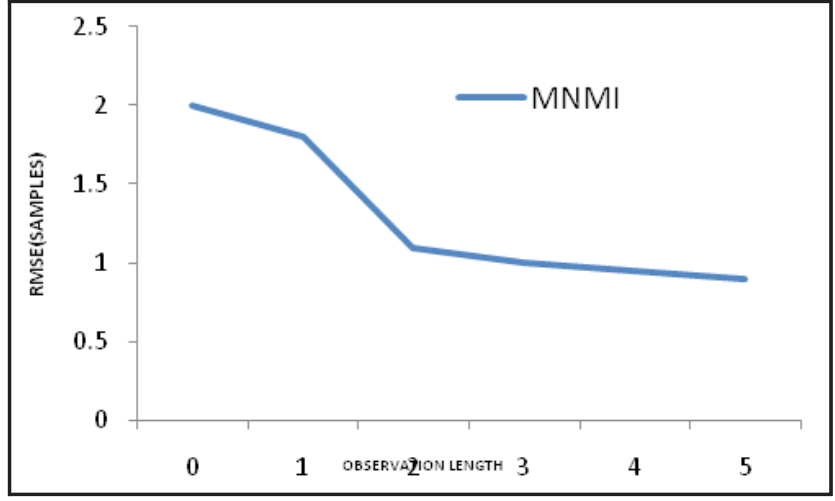

Figure 2. Observation length Vs RMSE (samples) for $\mathrm{SNR}=-20 \mathrm{db}$.

\section{Result and Conclusion}

In this paper a new time delay estimator in multipath environment is proposed. The spreading of signals in neighboring samples is used to estimate the time delay. Non mutual information criteria is used in this paper. Simulation results validate the effectiveness of the proposed algorithm. The future work could be to analyze the computational complexity of the proposed algorithm with the non mutual information based time delay estimator.

\section{References}

1. Gay SL, Benesty J. Acoustic signal processing for telecommunication. Kluwer Academic, 2000.

2. Knapp C, Carter GC. The generalized correlation method for estimation of time delay. IEEE Trans Acoust Speech Signal Process. 1976; 24(4):320-27.

3. Benesty J, Huang Y, Chen J. Time delay estimation via minimum entropy. IEEE Signal Process Lett. 2007, 14(3):157-60.

4. Cover TM, Thomas JA. Elements of Information Theory. John Wiley \& Sons; 2012.

5. Pluim JPW, Maintz JBA, Viergever MA. Mutualinformation-based registration of medical images: a survey. IEEE Trans Med Imag. 2003, 22(8):986-1004.

6. Matsuda, H. Physical nature of higher-order mutual information: Intrinsic correlations and frustration. Physical Review E. 2000. 2000; 62(3):3096.

7. Wen F, Wan Q. Robust time delay estimation for speech signals using information theory: A comparison study. EURASIP JASMP. 2011; 2011(1):1-10.

8. Talantzis F, Constantinides AG, Polymenakos LC. Estimation of direction of arrival using information theory. IEEE Signal Process Lett. 2005; 12(8):561-64. 
9. Chen J, Benesty J, Huang YA. Time delay estimation in room acoustic environments: an overview. EURASIP JASMP. 2006; 2006.

10. Nath R. Adaptive echo cancellation based on a multipath model of acoustic channel. Circ Syst Signal Process. 2013; 32(4):1673-98.

11. Chaturvedi AK, Sharma G. Parallel recursive least squares algorithms for adaptive Volterra filters. 1992 IEEE International Symposium on Circuits and Systems, ISCAS'92; 1992; IEEE.

12. Chaturvedi AK, Sharma G. A new family of concurrent algorithms for adaptive Volterra and linear filters. IEEE Trans Signal Process. 1999; 47(9):2547-551.

13. Nath R. Modified Generalized Autocorrelation based Estimator for time delays in multipath environmentA tradeoff in estimator performance and number of multipath. Comput Electr Eng. 2011; 37(3):241-52.

14. Moghaddam PP, Amindavar H, Kirlin RL. A new timedelay estimation in multipath. IEEE Trans Signal Process. 2003; 51(5):1129-42.

15. Carter GC, Nuttall AH, Cable P. The smoothed coherence transform. Proc IEEE. 1973; 61(10):1497-98.

16. Chen J, Benesty J, Huang Y. Robust time delay estimation exploiting redundancy among multiple microphones. IEEE Trans Speech Audio Process. 2003; 11(6):549-57.

17. $\mathrm{He}, \mathrm{H}$ et al. Time delay estimation via non-mutual information among multiple microphones. Appl Acoust. 2013; 74(8):1033-36.

18. Srinivasa S. A review on multivariate mutual information. Univ. of Notre Dame, Notre Dame, Indiana; 2008.

19. de Godoy JC, Rodrigo VAOA, Garcia C. Time delay: an alternative definition for optimal system identification.
2013 IEEE 52nd Annual Conference on Decision and Control (CDC); 2013; IEEE.

20. Chien Y-R, Chu S-I. A fast converging partial update lms algorithm with random combining strategy. Circ Syst Signal Process. 2014:1-16.

21. Mahbub Upal et al. Single-channel acoustic echo cancellation in noise based on gradient-based adaptive filtering. EURASIP JASMP. 2014; 2014(1):20.

22. Nath R, Chaturvedi AK, Sharma G. Blind estimator for time delays in multipath acoustic channel? estimator performance vs. noise and number of multipath. Int J Signal Imag Syst Eng. 2011; 4(1):13-20.

23. Song A, Qiu T. The equivalency of minimum error entropy criterion and minimum dispersion criterion for symmetric stable signal processing. IEEE Signal Process Lett. 2010; 17(1):32-35.

24. Vicente R, Wibral M. Efficient estimation of information transfer. Directed Information Measures in Neuroscience. Springer Berlin Heidelberg; 2014.

25. Petsatodis T et al. Exploring super-gaussianity toward robust information-theoretical time delay estimation. J Acoust Soc Am. 2013; 133(3):1515-24.

26. Hennecke $M H$, Fink GA. Robust multichannel acoustic time delay estimation in reverberant environments. ITGFachbericht-Sprachkommunikation. 2010; 2010.

27. Liu Q, Han SW, Ma XS. Research of time delay estimation based on higher order statistics. Appl Mech Mater. 2014; 530:550-53.

28. Ying L, Jianping L, Yiwen Z. Minimum entropy-based acoustic source localization with Laplace Distribution. 2010 IEEE 10th International Conference on Signal Processing (ICSP); 2010; IEEE. 